\title{
PARAS-2 precision radial velocimeter: optical and mechanical design of a fiber-fed high resolution spectrograph under vacuum and temperature control
}

Abhijit Chakraborty, Nitesh Thapa, Kapil Kumar, Prasad J. S. S. V. Neelam, Rishikesh Sharma, et al.

Abhijit Chakraborty, Nitesh Thapa, Kapil Kumar, Prasad J. S. S. V. Neelam, Rishikesh Sharma, Arpita Roy, "PARAS-2 precision radial velocimeter: optical and mechanical design of a fiber-fed high resolution spectrograph under vacuum and temperature control," Proc. SPIE 10702, Ground-based and Airborne Instrumentation for Astronomy VII, 107026G (20 July 2018); doi: $10.1117 / 12.2313055$

SPIE Event: SPIE Astronomical Telescopes + Instrumentation, 2018, Austin, Texas, United States 
PARAS-2 precision radial velocimeter: optical and mechanical design of a fiber-fed high resolution spectrograph under vacuum and temperature control

\author{
Abhijit Chakraborty ${ }^{1 *}$, Nitesh Thapa ${ }^{2}$, Kapil Kumar ${ }^{1}$, Neelam J. S. S. V. Prasad ${ }^{1}$, Rishikesh Sharma ${ }^{1}$, \\ Arpita Roy ${ }^{3}$ \\ ${ }^{1}$ Astronomy \& Astrophysics Division, Physical Research Laboratory, Ahmedabad, 380009, India \\ ${ }^{2}$ Space Applications Centre, Ahmedabad, 380015, India \\ ${ }^{3}$ Department of Astronomy, California Institute of Technology, Pasadena, CA 91125, US \\ *abhijit@prl.res.in; phone +91-79-26314607
}

\begin{abstract}
We present here the optical and mechanical design of a fiber-fed High-resolution spectrograph at resolution $(R)=100,000$ which will be under vacuum (0.001 to 0.005 mbar) and temperature controlled environment at $25 \mathrm{C} \pm 0.001 \mathrm{C}$. The spectrograph will be attached to our upcoming new PRL $2.5 \mathrm{~m}$ aperture telescope at Gurushikar, Mount Abu, Rajasthan, India. The spectrograph is named PARAS-2 after the successful operation of PARAS (PARAS-1) with our existing $1.2 \mathrm{~m}$ aperture telescope at Gurushikar, Mount Abu since 2012 summer. The spectrograph (PARAS-2) will be operating in the range of $380 \mathrm{~nm}$ to $690 \mathrm{~nm}$ wavelength in a single shot using Grism as a Cross Disperser, R4 Echelle at blaze angle of 76degrees, and pupil diameter of $200 \mathrm{~mm}$. We will use a combination of octagonal and circular fibers along with double scrambler and simultaneous calibration for getting down to the RV precision of $50 \mathrm{~cm} / \mathrm{s}$ or better $(<50 \mathrm{~cm} / \mathrm{s})$. Minimum $30 \%$ time will be reserved for exoplanet work with the spectrograph on the $2.5 \mathrm{~m}$ telescope when it becomes operational in early 2020 . The overall efficiency of the whole spectrograph (Echelle, M1, M2, FM, Grism, Camera lens system, Dewar window) excluding fiber is expected to be $22.5 \%-28 \%$ and $4 \%-8 \%$ including optical fiber, telescope and fibertelescope interface losses.
\end{abstract}

Keywords: High resolution Spectroscopy, Echelle, Grism, Exoplanets

\title{
1. INTRODUCTION
}

The precise Doppler velocity measurements or velocimetry measure star's wobble around its axis and determine the mass and the orbital parameters (like period, semi-major axis, eccentricity, etc.) of the planet going around it. This is the most reliable technique to detect and characterize the mass of exoplanets from ground-based telescopes. The increasing population of small size planets (superEarths) detected by space-based transit photometry missions (like CoRoT, Kepler, K2 and upcoming TESS, PLATO) has opened an opportunity among astronomers around the world to build very precise high-resolution spectrographs to determine their masses and orbital parameters. $50 \mathrm{~cm} / \mathrm{s}$ or better precision is essential to detect or characterize massive or super-Earths around $\mathrm{G}$ and $\mathrm{K}$ dwarf (Sun-like) stars. Further, Exoplanet research requires lots of dedicated telescope time, which is impossible to get on large telescopes. Therefore, small or moderate telescopes of apertures $2.5 \mathrm{~m}-4 \mathrm{~m}$ can be efficiently used for exoplanet research.

Ground-based and Airborne Instrumentation for Astronomy VII, edited by Christopher J. Evans, Luc Simard, Hideki Takami, Proc. of SPIE Vol. 10702, 107026G · C 2018 SPIE · CCC code: 0277-786X/18/\$18 · doi: 10.1117/12.2313055 
Physical Research Laboratory (PRL) started an exoplanet program (Chakraborty et al. 2008) in 2008, with PARAS (PRL Advanced Radial Velocity Abu Sky Search, R 67,000), where we have consistently demonstrated 3 to sub $2 \mathrm{~m}$ stability (Chakraborty et al. 2014, 2018) on bright standard RV stars.

We are now developing a new fiber-fed high-resolution spectrograph, which will be able to achieve RV measurements of $50 \mathrm{~cm} / \mathrm{s}$ or better $(<50 \mathrm{~cm} / \mathrm{s})$. As per the science requirements PARAS- 2 is to cover the full spectral range at one shot covering Echelle orders from 90 (690nm wavelength) to 162 (380nm wavelength). We present here the design concept of this new high-resolution $(R=100,000)$ optical fiber-fed Echelle spectrograph. The spectrograph will be coupled with the PRL $2.5 \mathrm{~m}$ telescope (which is expected to see first light in early 2020). The spectrograph is called PARAS-2 as it is the successor of the spectrograph PARAS (now being called as PARAS-1). Our primary motivation is to target super-Earths and super-Neptunes ( 2 to 40 MEARTH) around $G$ \& $K$ dwarfs, and to achieve long-term stability of 50 $\mathrm{cm} / \mathrm{s}$ on bright stars (up to 7th magnitude), sub $2 \mathrm{~m} / \mathrm{s}$ (up to 10th magnitude), and sub $5 \mathrm{~m} / \mathrm{s}$ (up to 12th magnitude).

The Optical design is presented in section 2 and mechanical stability of the spectrograph in section 3 . Details of fiber optics interface and thermal stability of the spectrograph are presented in section 4 and 5 , respectively. We summarize the work in section 6 .

\section{PARAMETERS CONSIDERED FOR OPTICAL DESIGN}

We have considered the following parameters while designing the spectrograph:

a) The spectrograph pupil diameter of size $200 \mathrm{~mm}$ ( 8 inches) with equivalent slit width of $166 \mu \mathrm{m}$ (the fiber diameter is 75 microns which projects an image size of 150 microns at the slit position through $\mathrm{F} / 3.6$ to $\mathrm{F} / 8$ transfer optics), b) high resolution $\left(\mathrm{R}^{\sim 100,000)}\right.$ and high spectrograph efficiency from $380 \mathrm{~nm}-690 \mathrm{~nm}$ and white pupil configuration, c) Grism cross disperser, large inter-order spacing for simultaneous referencing without cross talking or interference between the spectra of same orders, d) mechanical stability, e) an adequate factor of safety over stress developed due to external pressure, f) Optical scrambling, and g) thermal stability.

\subsection{A brief description of the optical design}

Figure 1 shows the complete optical layout of the Echelle spectrograph, which is in white pupil configuration with grism as cross-disperser. Through optical design optimization, we conclude that minimal distortion and highly stable PSF (point spread function corresponding to fiber illumination) are requisite to achieve sub-meter Radial Velocity precision on stars. M1 and M2 are two off-axis parabolic mirrors of the radius of curvature of $3200 \mathrm{~mm}$ cut from a single circular glass piece of diameter 800 $\mathrm{mm}$. The physical size of M1 is $260 \mathrm{~mm} \mathrm{X} 400 \mathrm{~mm}$ and that of M2 is $240 \mathrm{~mm} \times 400 \mathrm{~mm}$. M1 is a segment of the parabola centered $165 \mathrm{~mm}$ below the apex of the parent parabola and $\mathrm{M} 2$ is a segment of the parabola centered $200 \mathrm{~mm}$ above the apex. M1 and M2 are placed on the optical bench at their respective places using rigid supports. 


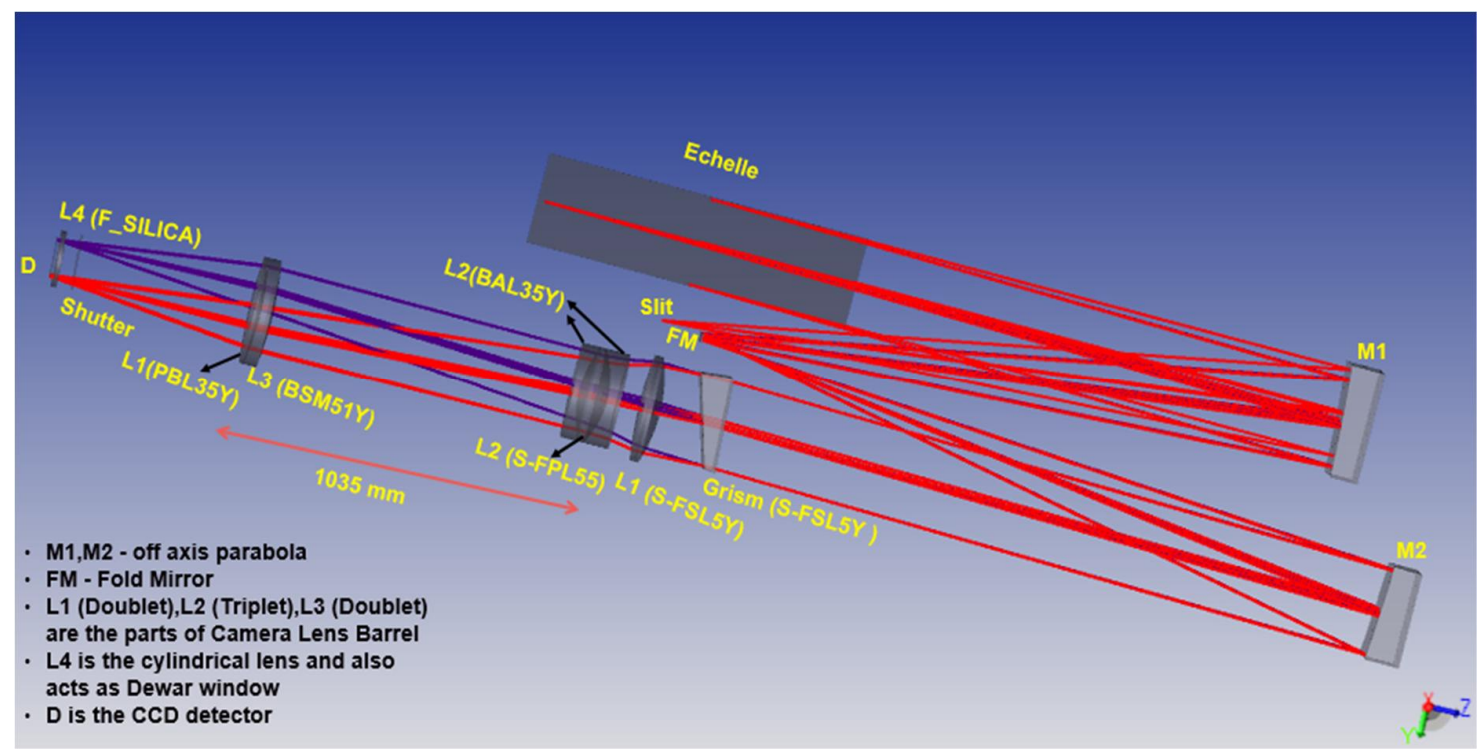

Figure 1. 3D Optical layout of the full spectrograph. Material chosen for optical elements is written in parentheses. The Red and Blue colored beams represent the lowest (90) and the highest optical orders (162), respectively.

The distance between the slit and the apex of the parent surface of M1 is $1600 \mathrm{~mm}$. The beam is collimated by $\mathrm{M} 1$ and reflected to the echelle. The size of the echelle is $840 \times 214 \times 125 \mathrm{~mm}$. The echelle grating has line frequency 31.6 grooves $/ \mathrm{mm}$ and blaze angle 76 degrees. It is from Richardson Gratings (Newport). The echelle is rotated by 76 degrees (blaze angle) about the $Y$-axis. The echelle is also tilted by 0.45 degrees about the $X$-axis to reflect back the light from the echelle at an angle with respect to the incident light and hence, after reflection by $M 1$ (its second reflection), the beam won't go back to the slit. Thus the light beam is reflected by M1, and reaches the Fold Mirror (FM), then after going through the intermediate focus it reaches the second off-axis parabolic segment, M2 (light path distance between FM and M2 is $1599 \mathrm{~mm}$ ). The flat fold mirror also folds the beam to save space and to accommodate the spectrograph in a reasonable space. The FM is deliberately kept away from the intermediate focus so that any dust particle sitting on the mirror surface or any other similar issues will not affect the PSF. After reflection by M2, the quasi-parallel light beam goes to the crossdisperser Grism (light path distance between M2 and Grism is $1600 \mathrm{~mm}$ ).

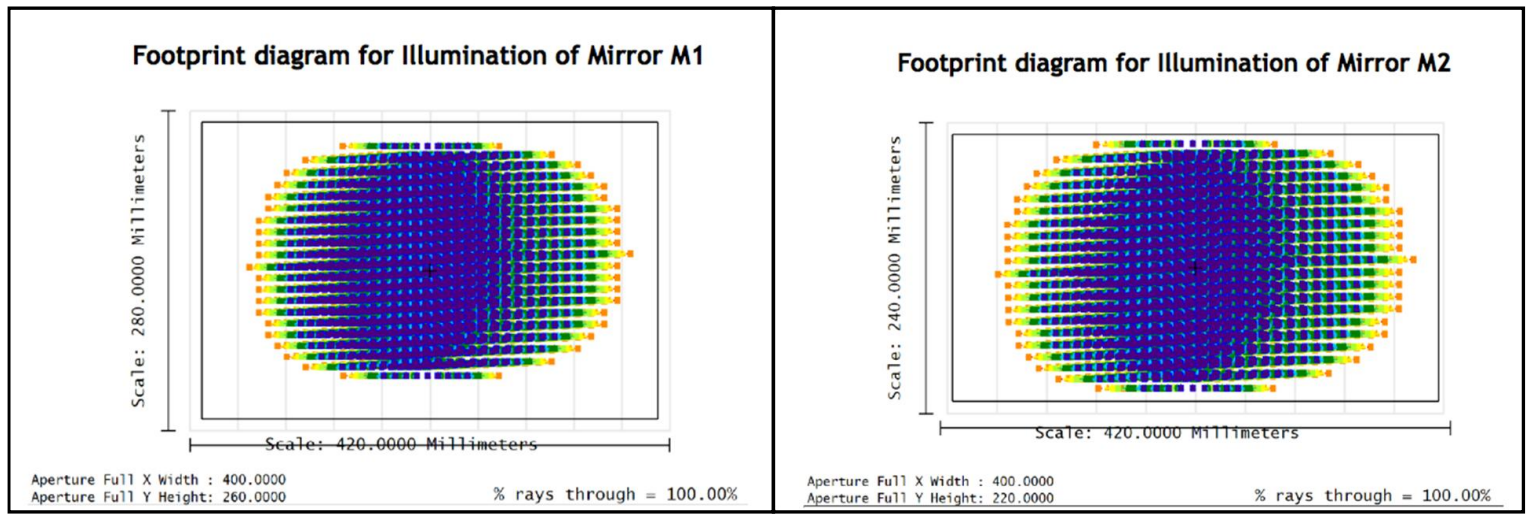

Figure 2a \& b. Footprint diagrams for Illumination of Mirror M1 (left) and Mirror M2 (right) after dispersion. 
The illuminated portion of the mirrors, $\mathrm{M} 1$ and $\mathrm{M} 2$, are shown in Figure $2 \mathrm{a} \& \mathrm{~b}$, respectively. Here the footprint shows the second pass on M1. A larger portion of the 1st mirror, M1, is illuminated after the grating disperses the light. The figure on the right shows the footprint on the M2 after the intermediate focus.

\subsection{Grism as cross disperser}

There are two options available for choosing the cross-disperser. It could be a single large prism or a grism. Though the throughput of the prism (80\%) is much higher than grism (65\%) over a wide range of wavelength $380 \mathrm{~nm}$ to $690 \mathrm{~nm}$, we have chosen grism over prism because of higher thermal stability of the custom designed grism. Grism has been designed such a way that the shift in the orders in cross disperser direction is minimal (see table 1, the Maximum shift is about $10 \mathrm{~nm}$ at temperature variation of $0.01 \mathrm{C}$, at $24 \mathrm{C}$ ). For the prism as cross disperser, our estimation shows that for similar thermal variation the shift of the spectra in cross-dispersion direction is of the order of a few $100 \mathrm{~s} \mathrm{of} \mathrm{nm}$.

Table 1: - Shift orders in cross-dispersion axis for temperature variation of $0.01^{\circ} \mathrm{C}$ at $24 \mathrm{C}$.

\begin{tabular}{|c|c|c|}
\hline Shift(nm) & $\begin{array}{c}\text { Wavelength } \\
(\mathrm{nm})\end{array}$ & Orders \\
\hline 9.066 & 679.60 & 90 \\
\hline 8.720 & 672.10 & 91 \\
\hline 7.729 & 650.70 & 94 \\
\hline 7.412 & 643.80 & 95 \\
\hline 5.925 & 611.60 & 100 \\
\hline 5.645 & 605.60 & 101 \\
\hline 4.077 & 571.60 & 108 \\
\hline 3.833 & 566.30 & 107 \\
\hline 2.462 & 536.50 & 114 \\
\hline 2.248 & 531.90 & 115 \\
\hline 0.672 & 497.30 & 123 \\
\hline 0.491 & 493.30 & 124 \\
\hline-1.140 & 456.40 & 134 \\
\hline-1.286 & 453.10 & 135 \\
\hline-2.692 & 418.90 & 146 \\
\hline-2.802 & 416.10 & 147 \\
\hline-3.944 & 382.30 & 161 \\
\hline-4.008 & 379.90 & 162 \\
\hline
\end{tabular}

Although this shift is in the cross-dispersion direction, this matters because of time elapse between the observed flats and the actual star observations. This affects the RV precision because of inaccurate extraction of the star spectra due to spectral drift. Moreover, PARAS-2 is designed to operate over a wavelength of $380 \mathrm{~nm}$ to $690 \mathrm{~nm}$ only, therefore the choice of grism over prism becomes technically more preferable as a cross-disperser. Here we have designed a single S-FSL5Y grism as a crossdisperser. As per the Data Sheet, glass S-FSL5Y (OHARA) has an internal transmission of 0.998 per 25 $\mathrm{mm}$. The Grism is made by Richardson Gratings (Newport). 
The axial thickness along the central ray is $50 \mathrm{~mm}$ with an apex angle of 13.24 degrees cutting short at the top thickness of $21.78 \mathrm{~mm}$. It is $74.88 \mathrm{~mm}$ thick at the base. The front face $(240 \mathrm{~mm} \mathrm{X} 240 \mathrm{~mm}$; Coated face diameter $=220 \mathrm{~mm}$ ) of the grism is AR coated (AR coating transmission $>99 \%$ between $380 \mathrm{~nm}$ to $690 \mathrm{~nm})$. The inclined face of grism has 257.14 lines $/ \mathrm{mm}$. The prism angle, the grating specification of lines $/ \mathrm{mm}$, and the chosen prism glass material in combination thus made it temperature variation insensitive at a level of $0.01 \mathrm{C}$ at $24 \mathrm{C}$.

\subsection{Glass material chosen for lenses}

Figure 3 shows the camera lens system consisting of four lenses (L1-doublet; L2-triplet; L3-doublet and L4 as fused silica Dewar window). The main conditions are that the lens system should have high internal transmission, and the final theoretical spot size should be within 15microns (EE80 diameter) with an F/number of around 5. The theoretical design was optimized for a spot size of EE80 diameter, which should be less than 15 microns (i.e. 1 pixel on the detector.) over the wavelength range of the spectrograph.

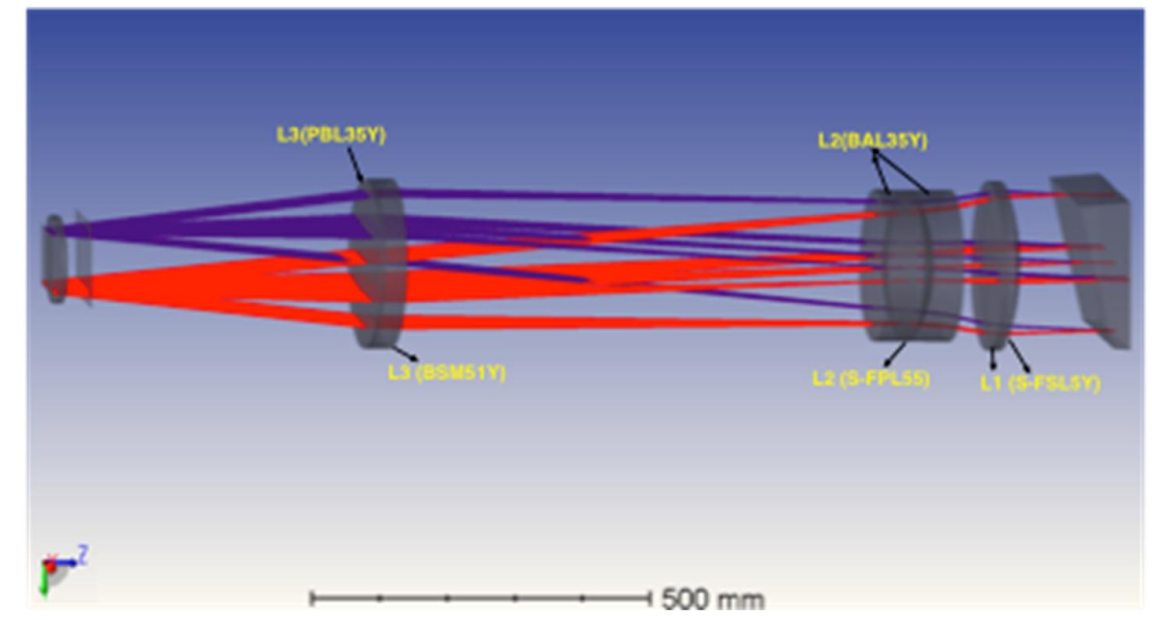

Figure 3. The F/5 Camera Optics 3D Layout separations in $\mathrm{mm}$. The blue and red colored beam represents here the highest and the lowest optical orders on the CCD detector, respectively.

L1, which is a singlet lens of glass S-FSL5Y (OHARA), has been made into a pseudo doublet with a split at $R 2$ for the ease of manufacturing the aspheric surfaces (conic constant of surface $1=0.4$, surface $2=-$ 3.9) under tight tolerances. The two halves will be joined by optical cement having optical properties similar to FK3 glass. The glass S-FSL5Y (OHARA) has the internal transmission of $99.8 \%$ per $25 \mathrm{~mm}$ of thickness as per data sheet. Therefore, the total transmission through L1 is $99.6 \%$. L2 is a triplet to be made in three spherical lenses, which will be joined together using optical cement having properties similar to FK3. The first and the third lenses are made of glass BAL35Y and the middle lens is made of glass S-FPL55Y. The glass BAL35Y has an internal transmission of $99.8 \%$ per $25 \mathrm{~mm}$ of thickness, therefore, the total internal transmission through this triplet is $99.4 \%$. L3 is a doublet made up of a spherical and an aspherical lens of glass material BSM51Y and PBL35Y respectively (L3 last surface conic constant $=-5.4$ ). The internal transmission of BSM51Y and PBL35Y is $99.8 \%$ per $25 \mathrm{~mm}$ of thickness. The total internal transmission through L3 is $99.6 \%$. Since all the six surfaces of lens system exposed to air or vacuum, are to be AR coated (Transmission=98.6\%), the overall transmission of the 
whole camera lens assembly is expected to be $91 \%$.

\subsection{Detector}

PARAS-2 spectrograph will have a back-thinned CCD detector consisting of 6144 X 6190 pixels from E2V (a CCD231-C6 device). This CCD has $15 \mu \mathrm{m}$ square pixels and E2V "Astro-broadband" antireflection (AR) coating that provides high quantum efficiency (QE) over most of the operating wavelength region $(380 \mathrm{~nm}-690 \mathrm{~nm})$. The measured quantum efficiency of the array is: $94.2 \%$ at $400 \mathrm{~nm}, 92.1 \%$ at $500 \mathrm{~nm}$, and $92.8 \%$ at $650 \mathrm{~nm}$.

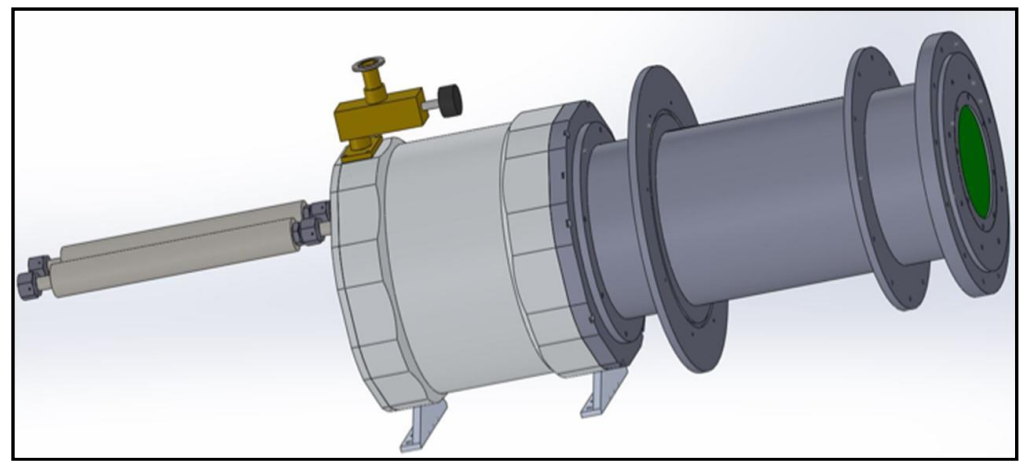

\section{Figure 4: Custom made CCD Dewar from IR Labs}

The back-thinned device does not exhibit any significant fringing at wavelengths between 600 to $690 \mathrm{~nm}$ where it is less than $1 \%$, making the standard coating sufficient for our purpose. The CCD Dewar and cryogenic assembly as shown in figure 4 is built by Infrared Laboratories, USA. We will be using Liquid Nitrogen $\left(\mathrm{LN}_{2}\right)$ for cooling the $\mathrm{CCD}$ to $-115^{\circ} \mathrm{C}$ and a temperature controller will be used to maintain the $\mathrm{CCD}$ at its optimum operating temperature of $-115^{\circ} \mathrm{C}$. The $\mathrm{CCD}$ control and data acquisition are accomplished with an SDSU Gen-4 Leach controller and associated electronics. Figure 5 shows the final spots (PSF) on the detector Image Plane. A computer running Microsoft Windows 10 and Owl software (supplied with the SDSU controller) will be used to acquire and display the data. The CCD exhibits a dark noise of $\sim 0.16$ electrons/pixel/hr at the standard operating temperature, and the slow-readout mode $(50 \mathrm{KHz})$ of the controller yields a read noise as low as 3 to $4 \mathrm{e}$, as per the data sheet provided by E2V. Figure 6 shows the footprint diagram of the CCD.

Spots at Echelon Blaze peaks of various orders

wavelength in $\mathrm{nm} \quad 679.6$ 672.1650.7 643.8611 .6605 .6571 .6566 .3536 .5531 .9497 .3493 .3456 .4453 .1418 .9416 .1382 .3379 .9

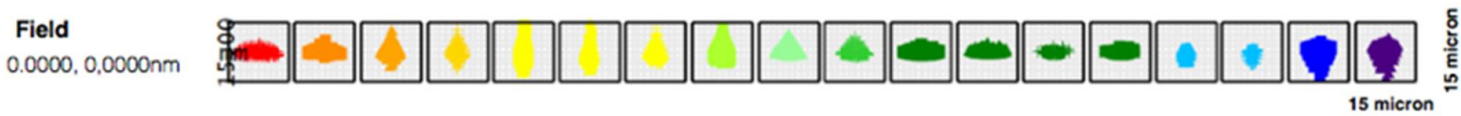

Figure 5. Spots defining PSF at the Detector at various wavelengths. 15micron is the pixel size on the detector. 


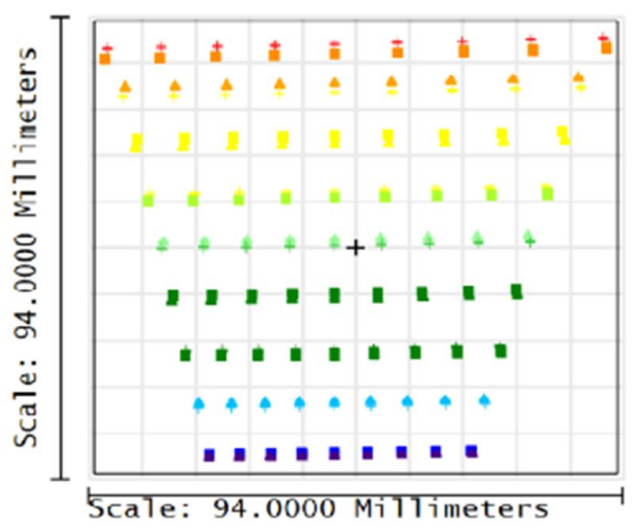

Aperture Ful1 $\times$ width : 92.0000

Anerture Ful1 Y Heicht: 92.0000

Figure 6: The footprint diagram for CCD detector. Total no. of orders on the detector is 18 . The lowest and highest optical orders are represented in red and blue color, respectively.

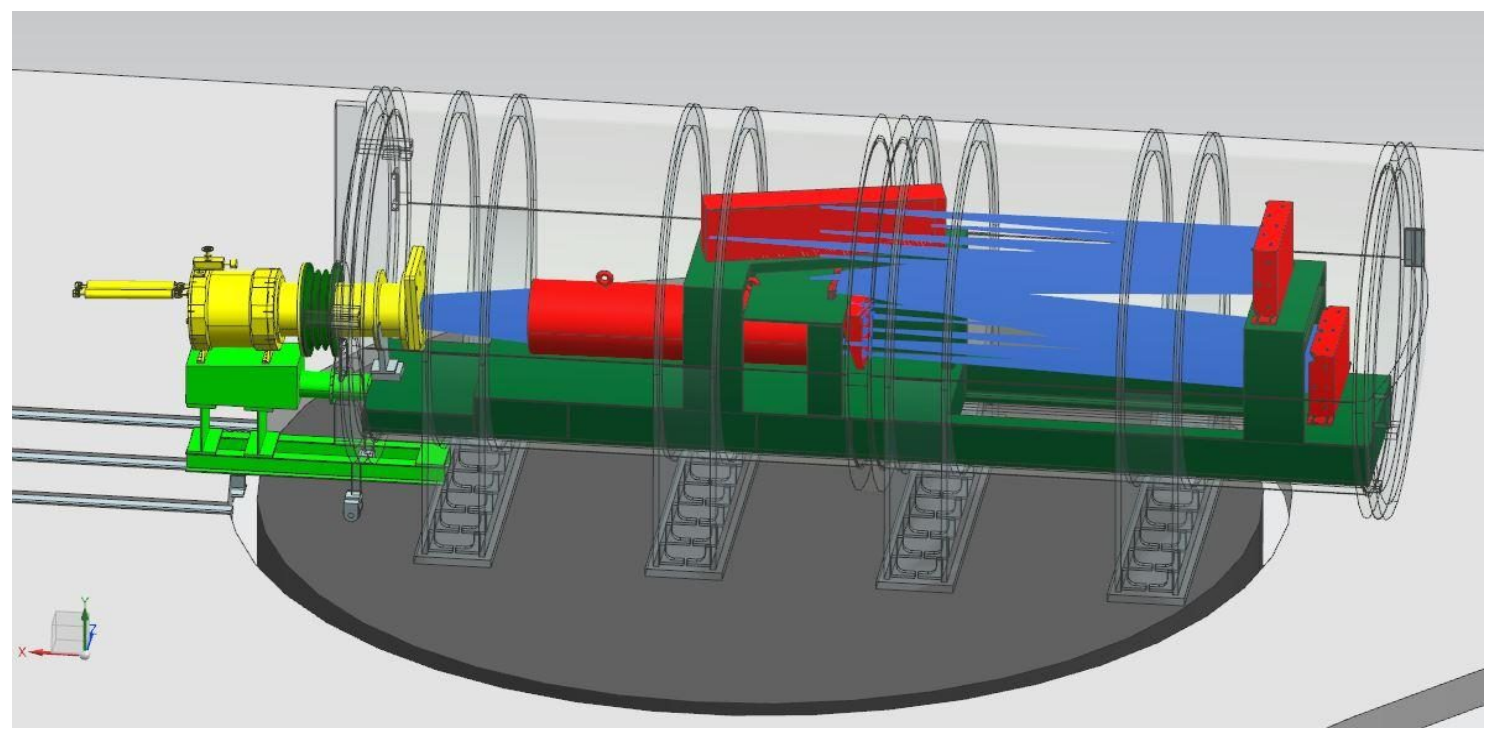

Figure 7: 3D View of PARAS-2 Vacuum Chamber kept over a dynamically isolated pier. Ray tracing through different optical element inside vacuum chamber is shown. CCD Dewar connection with vacuum chamber with the bellow is also shown.

The average efficiency from the slit position to the detector is found to be 22.5 - $28 \%$ on considering greater than $95 \%$ reflectivity for the three mirror surfaces, 0.65 for Echelle blaze peak efficiency, 0.55 to 0.70 for grism efficiency, and 0.9 QE for the CCD array. 


\section{Mechanical Stability}

PARAS-2 optical elements (Echelle, Off-axis parabolas, lens barrel etc.) will be arranged on a very stiff optical bench (see figure 7) through their respective support system and in a vacuum and temperature controlled environment. Theoretically, the spot sizes are designed to be within the single pixel size of the detector (15-micron square) over the entire wavelength region, so that maximum degradation of PSF, after aligning the optics, does not exceed $\mathbf{1 . 7}$ pixels or up to $\mathbf{2 5}$ microns. This whole assembly will be kept inside a cylindrical vacuum vessel. Optical bench inside vacuum vessel is supported over side extrusions in such a way that most of the weight of the optical bench comes over stiffening rings. There will be a linear bearing system installed below the optical bench to move it, in and out of the vacuum vessel. The whole vacuum vessel will be kept over RCC pier through clamped four saddle supports.

The maximum von-Mises stress generated is around $16 \mathrm{Mpa}$ at the center of the back lid. The material chosen to fabricate vacuum vessel and optical Bench is of SS-304 vacuum grade. From the material catalogues, the yield strength of SS-304 is about $206 \mathrm{Mpa}$. This gives an adequate factor of safety over the stress of about 13 . Figure 8 shows the deformations distribution over the entire vacuum vessel. The maximum deformation is around 104 microns at the back lid. Deformations over front lid are critical because it is connected with CCD Dewar extended tube and thus it will affect the optical alignment. Over this region, maximum deformations are up to 85 microns.

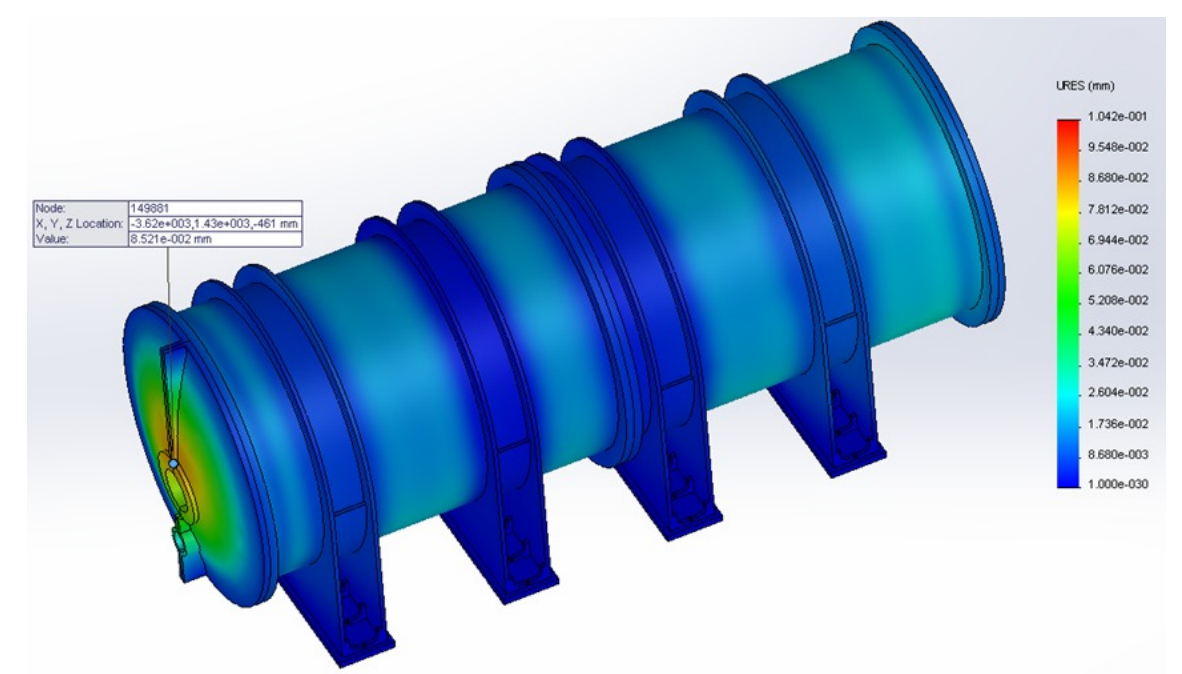

Figure 8: Deformations distribution over vacuum vessel due to pressure difference of 1atm

The 85 micron deformation is further taken care through the Metallic Bellow, coupling the Dewar with the vacuum chamber. The front side of the Dewar where CCD is installed will be clamped to the optical bench for rigidity and backside of the Dewar is simply rested on the bench outside the vacuum chamber for stress relieving. The bellow serves two purposes; a) It gives a margin in linear and angular adjustment during optical alignment $b$ ) It absorbs the deformations in vacuum vessel front lid due to external pressure hence leaving E2V sensor undisturbed which is kept at the end of the extended tube of CCD Dewar as shown in figure 7. The vacuum chamber will be kept over an RCC pier, which is dynamically isolated from rest of the facility. The Vacuum chamber will be made by AHV, Ahmedabad. 


\section{Fiber-Optic-Spectrograph interface}

In PARAS 2, we will use two $75 \mu \mathrm{m}$ diameter octagonal optical fibers from the telescope focal-plane unit. One fiber will carry the starlight from the telescope and the other, the calibration source light from the Thorium-Argon (ThAr) Calibration Spectral Lamp (Lovis et. al 2007) or Fabry Perot. These are coupled with FBP circular fibers through double scrambler (Hunter \& Ramsey et al. 1992). The octagonal and circular Fiber is being made by Polymicro Technologies (Molex). The two circular $75 \mu \mathrm{m}$ diameter core fibers at the spectrograph end will be separated by $250 \mu \mathrm{m}$ (using dummy fibers in a fiber bundle) and are re-imaged at the slit by the combination of two doublets (F/3.6 to F/8). The $250 \mu \mathrm{m}$ separation corresponds to a 22-pixel separation on the detector. This is the optimal separation required between the star and ThAr (or calibration) spectra for proper radial velocity estimation without the cross talk or interference between the two spectra.

Median seeing in winters at Mount Abu is around 1.2" and in summers it is around $1.4^{\prime \prime} .75 \mu \mathrm{m}$ core fiber at the $\mathrm{F} / 4$ focal plane of the $2.5 \mathrm{~m}$ telescope is about $1.5^{\prime \prime}$. This will allow $~ 75 \%$ of the light into the fiber when seeing is around 1.4". Further, we will also use a Tip/Tilt mechanism (see figure $9 a \mathrm{ab}$ ) at a rate of up to $20 \mathrm{~Hz}$, which will make the core of the star PSF further tighten to close to $1^{\prime \prime}$, making $>85 \%$ of the light injected into the fiber. The telescope tertiary (M3) mirror, which will be a Tip/Tilt mirror, will be used both as on-axis guiding and as well as for first-order seeing corrections to 1" (one arcsec). Starlight from M3 mirror (Tip/Tilt) will split into two beams by the beam splitter kept at 45degree (in between M3 and ADC where ADC is Atmospheric Dispersion Corrector). $10 \%$ of the star light will go to the Tip/Tilt and Auto-Guider camera that is Zyla 4.2 ANDOR CMOS camera which has 100fps with camera link (M3 mirror Tip/Tilt has to be corrected at a rate of up to $20 \mathrm{~Hz}$ So camera frame rate should be much faster). The $2.5 \mathrm{~m}$ telescope is from AMOS, Belgium.
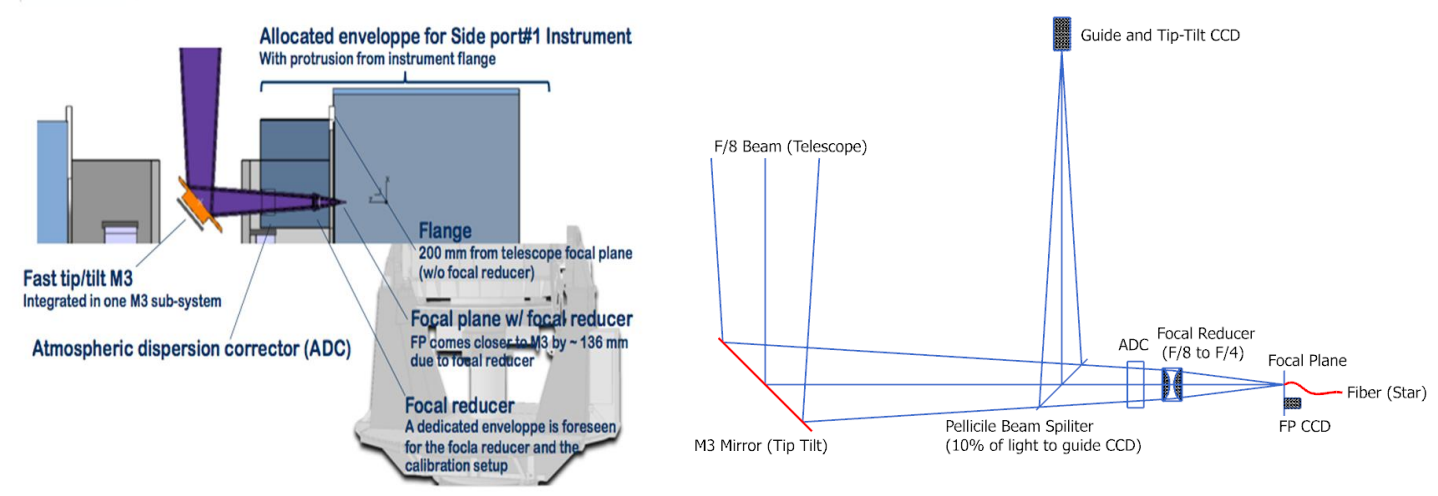

Figure 9a \& b. Input optics scheme for the starlight injection onto the fiber tip

This camera will be able to guide the stars brighter than $14^{\text {th }}$ magnitude with a tracking accuracy of $0.1^{\prime \prime}-0.2^{\prime \prime}$ over a period of one hour. Remaining $90 \%$ of the starlight will pass through the ADC and Focal Reducer ( $F / 8$ to $F / 4, \sim 1.5$ " on 75 micron fiber tip). Two precision actuators ( $x$-axis and $y$-axis) from Physik Instruments (PI) will be used to move the mechanical arm holding the fiber tip in the telescope focal plane with a precision better than $3 \mu \mathrm{m}$. The arm is also equipped with a small CCD camera (Lodestar-X2 CCD from Starlight- Xpress- Focal Plane CCD). The PI actuators first move the Focal plane 
CCD camera into the telescope beam, then star focus is checked. Next, the fiber tip is moved into the position of the CCD camera. The calibration fiber will also be on a PI actuator with choice of either seeing the Calibration spectra or sky back ground (not star light). Note that we have not shown the path of calibration light on to the star fiber through a flip mirror in Figure 9a or b. There will be three observational modes for the PARAS-2 spectrograph: 1) Acquisition of only star's spectra, 2) Simultaneous acquisition of star and ThAr spectra for continuous wavelength calibration during the exposure (Mainly used for extra-solar planet searches around stars) and 3) star's spectra along with sky spectrum for background subtraction.

\section{Thermal Stability}

Temperature variation by $1^{\circ} \mathrm{C}$ or Atmospheric pressure variation by 1 mbar would produce effects of the order of $100 \mathrm{~m} / \mathrm{s}$ (Pepe et al. 2002). The optical elements of the PARAS-2 instrument are ultrasensitive to temperature and pressure. Therefore, it becomes necessary to house the instrument optics in a very stable temperature and pressure controlled environment as shown in figure 10b. The vacuum chamber will be kept at a constant pressure of 0.001 to $0.005 \mathrm{mbar}$. The outer room is maintained at a lower temperature than the inner room to control the temperature in the vacuum chamber with an accuracy of $0.001^{\circ} \mathrm{C}$ RMS.

Heating can be controlled more efficiently than cooling. For PARAS-1 (Chakraborty et al. 2014), we have achieved temperature stability very close to the desired goal by means of enclosing PARAS-1 spectrograph in a closed concentric volume space consisting of two thermally insulated outer and inner chambers (see figure 10a).
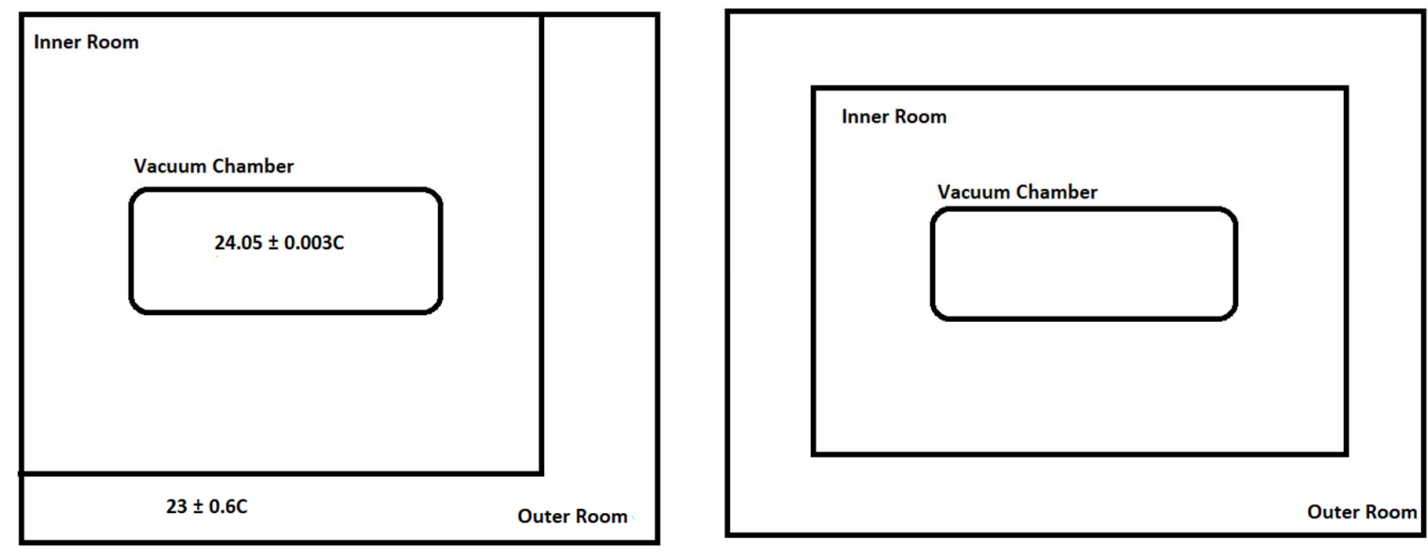

Figure 10a \& b: Schematic Layout of PARAS-1 (Left) and Proposed Layout of PARAS-2 (Right).

The outer room temperature control is achieved at $23 \pm 0.6^{\circ} \mathrm{C}$ by conventional heating and cooling algorithm (PID controlled). Vacuum chamber temperature in the inner room is maintained by using PRL made temperature controller. Vacuum chamber set point is $24.05^{\circ} \mathrm{C}$. It takes about 10 hours of time for a raise of $1^{\circ} \mathrm{C}$, this is done by heating the nichrome wire pads located on the walls of the inner chamber. This can be controlled by using Microcontroller and Power supply. We are using Lakeshore 336 for temperature reading from the sensor. These temperature values are sent to MCU through LabVIEW and serial communication. The MCU Unit compares measured values with the set point and gives an appropriate command to the power supply. Thus, the temperature of the spectrograph is 
controlled. Figure 11a and $\mathrm{b}$ show the temperature results we got from PARAS-1.
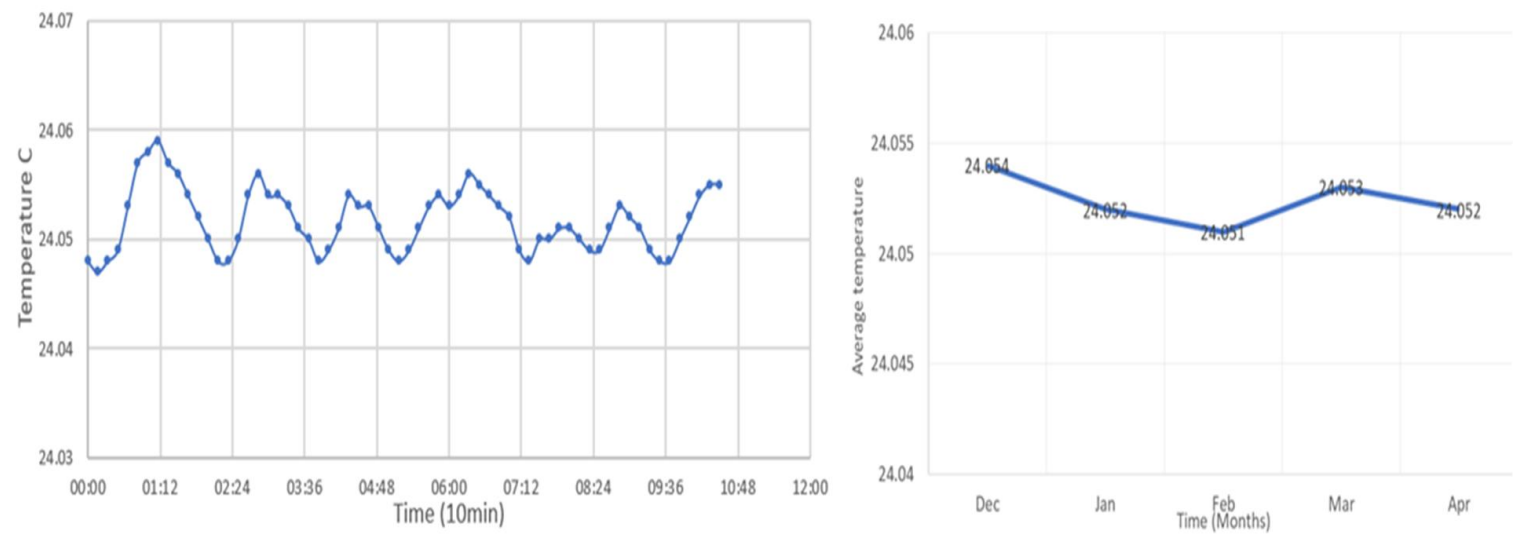

Figure 11a \& b: RMS value for a typical observing night is $0.003^{\circ} \mathrm{C}$ and peak to valley is $0.012^{\circ} \mathrm{C}$. Variation of average temperature throughout the months for one observing semester (Dec 2016 to April 2017)

For PARAS-2, we will use pt100 sensors with an accuracy of $0.001^{\circ} \mathrm{C}$ along with MicroK (from ISOTECH), which makes temperature measurement more accurate. Figure 12a shows the block diagram of the proposed temperature controller of PARAS-2. A total of 12 pt100 sensors will be used. 6 of them will be kept next to optics (Grism, Echelle, M1, M2, Fold Mirror and Camera lens) to measure the temperature. Heater pads will be kept on the floor of the inner room at a distance of $1.5 \mathrm{~m}$ away from the vacuum chamber. Another 6 sensors will be used to control the inner room temperature at a precision of $0.01^{\circ} \mathrm{C}$ at $24^{\circ} \mathrm{C}$.

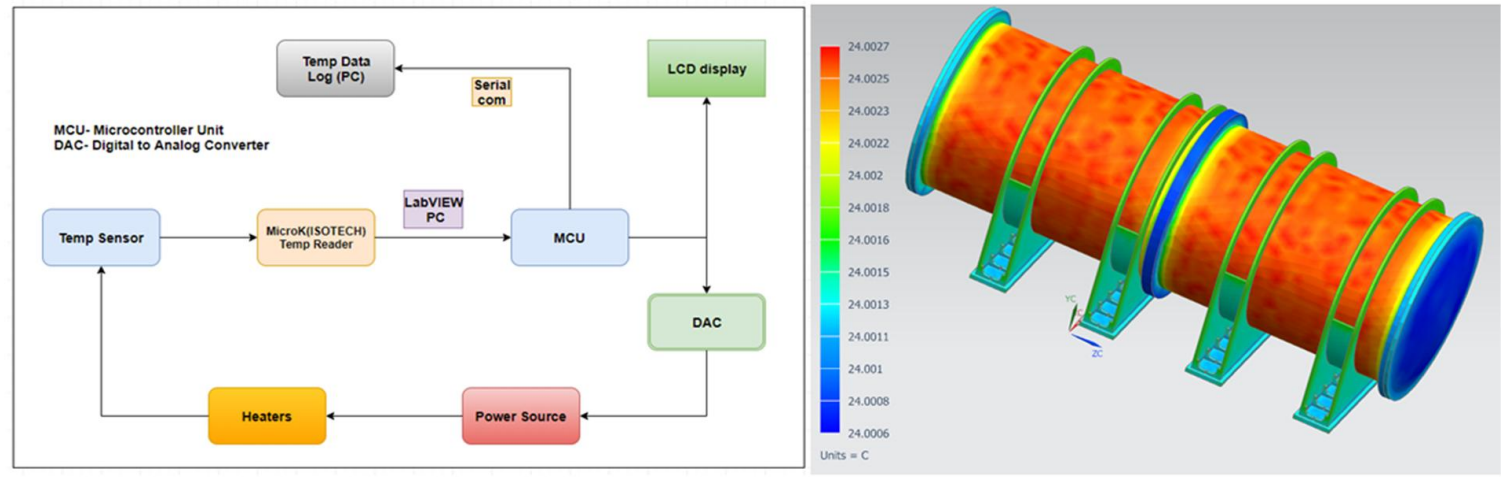

Figure 12a \& b: Block Diagram of Proposed Temperature Controller of PARAS-2; Simulation of Temperature distribution over entire vacuum chamber (0.002C PV) for a change of $0.01 \mathrm{C}$ in inner room.

Our simulation (See figure 12b) show that due to the thermal inertia of the vacuum chamber mass, the temperature variation across vacuum chamber is only $0.002^{\circ} \mathrm{C}$ (peak to valley) for a change of $0.01^{\circ} \mathrm{C}$ across the inner room. Heater pads will also be applied with thermal control at RCC Pier junction and at LN2 fill tubes and Dewar portion area to keep inner room temperature at $24^{\circ} \mathrm{C}+/-0.01^{\circ} \mathrm{C}$. 


\section{Summary}

We have presented here the optical and mechanical design concepts of an efficient fiber-fed highresolution echelle spectrograph PARAS-2, which is a white pupil configuration using R4 Echelle (blaze angle of 76degrees, Echelle mosaic of an area of $214 \mathrm{~mm} \times 840 \mathrm{~mm}$ ) and a single large Grism as Cross disperser. The spectrograph will work at a resolution of 100,000 . The spectrograph will be coupled with a $2.5 \mathrm{~m}$ telescope. The prime design consideration is to have one of the most precise spectrographs, achieving $50 \mathrm{~cm} / \mathrm{s}$ or better $(<50 \mathrm{~cm} / \mathrm{s})$. By proper choice of glass material, using a single large grism as cross disperser, anti-reflection coatings which will work at a high angle of incidence, and special UVenhanced mirror reflective coatings, we are expecting $22.5 \%-28 \%$ average efficiency from the slit to the detector. Thus the total efficiency of the spectrograph including fiber optics, telescope and the sky is expected to be around $4 \%$ to $8 \%$. It will be kept inside a vacuum chamber and under temperature controlled environment. The spectrograph will be primarily used for planet survey at an RV accuracy of $50 \mathrm{~cm} / \mathrm{s}$ for stars brighter than 7 th magnitude, sub $2 \mathrm{~m} / \mathrm{s}$ up to $10^{\text {th }}$ magnitude, and sub $5 \mathrm{~m} / \mathrm{s}$ up to $12^{\text {th }}$ magnitude. First light of the spectrograph will be in early 2020 .

\section{Acknowledgement}

This entire project is funded by PRL \& DOS, India. We are thankful to the PRL Director and DOS for support and funding. We also acknowledge the help from Francesco Pepe (Geneva Observatory) and Andrew Szentgyorgyi (Smithsonian Astrophysical Observatory, Harvard) for sharing the HARPS Grism design. We are thankful to Andrew Szentgyorgyi (SAO, Harvard) for reviewing the entire Spectrograph design and giving us very useful comments. The authors would also like to thank at PRL whose continuing valuable support has made this project possible. We acknowledge the help from Vishal Shah, Kevikumar Lad, Ashirbad Nayak from PRL, and D. Subramanyam (retired) from SAC, ISRO, India.

\section{References}

[1] Chakraborty, A., Richardson, E. H., \& Mahadevan, S. 2008, Proc. SPIE, 7014, 70144G

[2] Chakraborty, A., Mahadevan, S., Roy, A., et al. 2010, Proc. SPIE, 7735, 77354N

[3] Chakraborty, A., Mahadevan S., Roy A., et al. 2014, PASP, 126, 133

[4] Chakraborty, A., Roy A., Sharma R., et al. 2018, AJ, 156, 3

[5] Hunter T.R. \& Ramsey L.W., 1992, PASP, 104, 1244

[6] Johnson, J.A., Marcy, G.W., Fischer, D.A., et al. 2006, ApJ, 647, 600

[7] Lovis, C. \& Pepe, F. 2007, A\&A, 468, 1115

[8] Redman, S. L., Lawler, J. E., Nave, G., Ramsey, L. W., \& Mahadevan, S. 2011, ApJS, 195, 24

[9] Roy, A., Chakraborty, A., Mahadevan, S., et al. 2016, Proc. SPIE, 9908, 99086R 